\title{
Identity-Based Proxy Signcryption Protocol with Universal Composability
}

\author{
Huifang Yu $\mathbb{D}^{1},{ }^{1}$ Zhicang Wang, ${ }^{2}$ Jianmin $\mathrm{Li}^{3},{ }^{3}$ and Xinzhe $\mathrm{Gao}^{4}$ \\ ${ }^{1}$ School of Communication and Information Engineering, Xi'an University of Posts \& Telecommunications, Xi'an 710121, China \\ ${ }^{2}$ School of Automation, Xi'an University of Posts \& Telecommunications, Xi'an 710121, China \\ ${ }^{3}$ Meteorological Observatory of Qinghai Province, Xining 810001, China \\ ${ }^{4}$ School of Computer, Qinghai Normal University, Xining 810008, China
}

Correspondence should be addressed to Huifang Yu; yuhuifang@qhnu.edu.cn

Received 25 August 2018; Accepted 14 November 2018; Published 2 December 2018

Academic Editor: A. Peinado

Copyright (c) 2018 Huifang Yu et al. This is an open access article distributed under the Creative Commons Attribution License, which permits unrestricted use, distribution, and reproduction in any medium, provided the original work is properly cited.

\begin{abstract}
Proxy signcryption means that the proxy signcrypter obtains the delegate authorization from the original signcrypter and then signcrypts the specified message on behalf of the original signcrypter. In this paper, we construct an identity-based proxy signcryption protocol (IBPSP) based on the universally composable (UC) framework. In the random oracle model, we prove that this protocol has the semantic security under the gap bilinear Diffie-Hellman and computational Diffie-Hellman assumptions. At the same time, an ideal functionality of the identity-based proxy signcryption protocol is defined in the UC security framework, and we also prove the equivalence between the universally composable identity-based proxy signcryption protocol and its IND-CCA2 and UF-CMA security. Analysis shows this IBPSP has both low computation complexity and semantic security together with UC security.
\end{abstract}

\section{Introduction}

Proxy signature [1] has the authenticity of signature but cannot guarantee the confidentiality of message. Gamage et al. [2] proposed the first proxy signcryption scheme, which can simultaneously realize two functions of public key encryption and proxy signature. Later, $\mathrm{Li}$ and Chen [3] constructed an identity-based proxy signcryption scheme by integrating identity-based cryptosystem and proxy signcryption, but this scheme does not meet the unforgeability and forward security. Chen et al. [4] devised a provably secure identity-based proxy signcryption scheme under the computational Diffie-Hellman and bilinear DiffieHellman assumptions. Ming et al. [5] constructed an identitybased proxy signcryption scheme without random oracles and proved its confidentiality and unforgeability. Zhou [6] devised secure identity-based generalized proxy signcryption without random oracles from bilinear pairings which has the properties of public verification in the proxy signcryption mode.
The UC security framework [7] can meet the requirement of modular design of protocols. The salient property of UC definitions of security is that they guarantee security even when a secure protocol is composed of an arbitrary set of protocols, or when the protocol is used as a component of an arbitrary system. It is an essential property in a complex and unpredictable environment. Moreover, UC definitions can guarantee security even when an unbounded number of protocol instances are carried out concurrently in an adversarially controlled manner; furthermore, they can guarantee nonmalleability with respect to arbitrary protocols. In the UC framework, a protocol is abstracted as an ideal functionality which is considered as a trusted third party (TTP) for specific tasks. Every player can deliver a command to the ideal functionality in a secure and authentic manner, and the ideal functionality executes the command according to its specification. Informally, one protocol can securely carry out a given ideal functionality if no adversary can obtain more advantages from an attack on an instance of real execution of the protocol than from an attack on an ideal process where the parties only submit their inputs to 
a TTP with appropriate functionality. In other words, it is required that an instance of real execution can be simulated in the ideal process. A protocol is said to securely realize ideal functionality if any effect caused by an adversary attacking the protocol can be achieved by an adversary attacking the ideal functionality. i.e., when devising the complex protocols, one can allow the involved parties to have secure access to ideal functionalities; when implementing the protocol, each ideal functionality is replaced by one protocol securely realizing the ideal functionality. Any ideal functionality can be UC realized as long as a majority of parties are supposed to be honest.

Canetti [8] corrected the ideal functionality definition of digital signature in 2001. Wang et al. [9] defined the ideal functionality of the identity-based signature protocol and simultaneously proved the equivalence between identitybased signature with UC security and classical identity-based signature with EUF-CMA security in an adaptive adversary model. Tian et al. [10] devised composable group communication and solved the composable security problem of multicast group communication. Tian et al. [11] devised secure multiparty computation with the universal composability and realized a fair and secure two-party computation protocol. Thus, they solved the fair and secure two-party computation problem, although Katz deemed that this problem could not be realized. Zhang et al. [12] devised a key exchange protocol and proved its security in the UC security framework. Yuan and Liu [13] devised a universally composable group ownership transfer protocol for RFID tags. Zhao et al. [14, 15] proposed a universally composable group signature protocol and group blind protocol. Li et al. [16] devised self-certified blind signcryption with UC security. Hu et al. [17] proposed a gateway-oriented password-authenticated key exchange protocol with universal composability.

Identity-based proxy signcryption is appropriate for applications in electronic governments and electronic commerce [18], such as online proxy auctions or contract signing by an authorized proxy signer. Consider an application such as a bank account owned by a busy boss. So as to withdraw money from their saving account, the boss must sign a withdrawal slip which can only be verified by the bank teller. In this case, when the boss is unable to sign personally, they can delegate their signing power to a proxy signer who can legitimately conduct transactions on their behalf. It is an interesting research problem of how to construct a secure identity-based proxy signcryption protocol applying to a more complex network environment. In this paper, we construct a novel identity-based proxy signcryption protocol (IBPSP) with universal composability, whose semantic security is based on the intractability of the gap bilinear Diffie-Hellman (gap BDH) and computational DiffieHellman $(\mathrm{CDH})$ problems. In the random oracle model, we prove its indistinguishability against adaptive chosenciphertext attacks (IND-CCA2) and existential unforgeability against adaptive chosen-message attacks (UF-CMA). At the same time, we define the ideal functionality of IBPSP and illustrate the equivalence between the universally composable IBPSP and its IND-CCA2 and UF-CMA security.

\section{Preliminaries}

In this section, we briefly introduce bilinear pairings and several computational problems together with the universally composable (UC) framework.

2.1. Bilinear Pairings. Let $G_{1}$ denote one cyclic addition group with prime order $q$ and let $G_{2}$ denote one cyclic multiplication group with the same prime order. In addition, $P$ is a generator of additive group $G_{1}$ and $e: G_{1} \times G_{1} \longrightarrow G_{2}$ is a bilinear map with the properties as below:

Bilinearity: $e(a U, b V)=e(U, V)^{a b}$ for any $U, V \in G_{1}$ and $a, b \in Z_{q}^{*}$

Nondegeneracy: there exists $U, V \in G_{1}$ such that $e(U, V) \neq 1_{G_{2}}$, where $1_{G_{2}}$ is the identity element of $G_{2}$

Computability: there is an efficient algorithm to calculate $e(U, V)$ for any $U, V \in G_{1}$

2.1.1. BDH Problem. Given $<P, a P, b P, c P>$ for any $a, b, c \in$ $Z_{q}^{*}$, the bilinear Diffie-Hellman $(\mathrm{BDH})$ problem is that it is computationally infeasible to determine the value of $e(P, P)^{a b c} \in G_{2}$.

2.1.2. $C D H$ Problem. Given $<P, a P, b P>$ for any $a, b \in Z_{q}^{*}$, the computational Diffie-Hellman $(\mathrm{CDH})$ problem is that it is computationally infeasible to determine the value of $a b P \in$ $G_{1}$.

2.1.3. $D B D H$ Problem. Given $<P, a P, b P, c P>$ for any $a, b, c \in Z_{q}^{*}$ and $\mathscr{X} \in G_{2}$, the decisional bilinear DiffieHellman $(\mathrm{DBDH})$ problem is to decide whether $e(P, P)^{a b c}=$ $\mathscr{X}$. If it holds, the DBDH oracle $\mathcal{O}_{D B D H}$ returns 1 ; else, $\mathcal{O}_{D B D H}$ returns 0 .

2.1.4. Gap BDH Problem. Given $<P, a P, b P, c P>$ for any $a, b, c \in Z_{q}^{*}$, the gap bilinear Diffie-Hellman (gap $\mathrm{BDH}$ ) problem is to determine the value of $e(P, P)^{a b c} \in G_{2}$ with the help of $\mathcal{O}_{\mathrm{DBDH}}$.

2.2. UC Framework. The UC framework [8] consists of a real-life model, ideal model, and hybrid model. In the UC framework, $\mathscr{Z}$ represents the external environment that consists of arbitrary protocols running concurrently with the given protocol. $\mathscr{Z}$ generates the inputs to all parties, reads all outputs, and interacts with the adversary in an arbitrary way throughout the computation. A protocol $\pi$ is said to securely realize an ideal functionality $\mathscr{F}$ if, for any real-life adversary $\mathscr{A}$ interacting with the protocol, there exists a simulator $\mathcal{S}$ such that no $\mathscr{Z}$ can distinguish with nonnegligible probability whether it interacts with $\mathscr{A}$ and parties running the protocol $\pi$ or with $\mathcal{S}$ and parties interacting with $\mathscr{F}$ in the ideal process.

Let $\operatorname{real}_{\pi, \mathscr{A}, \mathscr{Z}}(k, z, r)$ denote the output of $\mathscr{Z}$ when interacting with the adversary $\mathscr{A}$ and parties $P_{1}, \ldots, P_{n}$ running protocol $\pi$ on security parameter $k$, input $z$, and random input $r=r_{\mathscr{E}}, r_{\mathscr{A}}, r_{1}, \ldots, r_{n}\left(z\right.$ and $r_{\mathscr{E}}$ for $\mathscr{E}, r_{\mathscr{A}}$ for $\mathscr{A}$, and 
$r_{i}$ for party $\left.P_{i}\right)$. Let $\operatorname{real}_{\pi, \mathscr{A}, \mathscr{E}}(k, z)$ be the random variable describing real $_{\pi, \mathscr{A}, \mathscr{E}}(k, z, r)$ when $r$ is uniformly chosen. Here real $_{\pi, \mathscr{A}, \mathscr{Z}}$ is the ensemble

$$
\left\{\operatorname{real}_{\pi, \mathscr{A}, \mathscr{Z}}(k, z)\right\}_{k \in N, z \in\{0,1\}^{*}} .
$$

Let ideal $_{\mathscr{F}, \mathcal{S}, \mathscr{E}}(k, z, r)$ denote the output of $\mathscr{Z}$ after interacting with the adversary $\mathcal{S}$ and ideal functionality $\mathscr{F}$ on security parameter $k$, input $z$, and random input $r=r_{\mathscr{Z}}, r_{\mathcal{S}}, r_{\mathscr{F}}\left(z\right.$ and $r_{\mathscr{Z}}$ for $\mathscr{Z}, r_{\mathcal{S}}$ for $\mathcal{S}$, and $r_{\mathscr{F}}$ for $\mathscr{F})$. Let $\operatorname{ideal}_{\mathscr{F}, \mathcal{S}, \mathscr{Z}}(k, z)$ be the random variable describing ideal $_{\mathscr{F}, \mathcal{S}, \mathscr{E}}(k, z, r)$ when $r$ is uniformly chosen. Here ideal $_{\mathscr{F}, \mathcal{S}, \mathscr{Z}}$ is the ensemble

$$
\left\{\operatorname{ideal}_{\mathscr{F}, \mathcal{S}, \mathscr{Z}}(k, z)\right\}_{k \in N, z \in\{0,1\}^{*}} \cdot
$$

Definition 1 (indistinguishability). Two binary distribution ensembles $\mathrm{X}$ and $\mathrm{Y}$ are indistinguishable (written $\mathrm{X} \approx \mathrm{Y}$ ), if, for any $c \in N$, there exists $k_{0} \in N$ such that, for all $k>k_{0}$ and for all $a$, we have

$$
|\operatorname{Pr}(\mathrm{X}(k, a)=1)-\operatorname{Pr}(\mathrm{Y}(k, a)=1)|<k^{-c} .
$$

Definition 2 (UC simulation). Let $\mathscr{F}$ denote an ideal functionality and let $\pi$ denote a probabilistic polynomial time protocol. We say that $\pi$ securely realizes $\mathscr{F}$ if, for any adversary $\mathscr{A}$, there exists an ideal-process adversary $\delta$ such that, for any environment $\mathscr{Z}$, we have

$$
\operatorname{ideal}_{\mathscr{F}, \mathcal{S}, \mathscr{Z}} \approx \operatorname{real}_{\pi, \mathscr{A}, \mathscr{Z}} .
$$

Theorem 3 (universal composition). Let $\mathscr{F}$ and $\mathscr{G}$ be ideal functionalities. Let $\pi$ be a protocol in the $\mathscr{F}$-hybrid model and let $\rho$ be a protocol which securely realizes $\mathscr{F}$ in the $\mathscr{G}$-hybrid model. Then for any adversary $\mathscr{A}$ in the $\mathscr{G}$-hybrid model, there exists an adversary $\mathcal{S}$ in the $\mathscr{F}$-hybrid model such that, for any environment $\mathscr{Z}$, we have

$$
\operatorname{real}_{\pi, \mathcal{S}, \mathscr{Z}}^{\mathscr{F}} \approx \operatorname{real}_{\pi^{\rho}, \mathscr{A}, \mathscr{E}}^{\mathscr{G}} \cdot
$$

\section{Formal Definition of IBPSP}

3.1. Algorithm Model. A universally composable IBPSP consists of five probabilistic polynomial time algorithms: Setup, Extract, PKeyGen, ProxyS, and Uns. Moreover, it involves four parties: a private key generator (PKG), an original signcrypter with identity $I_{a}$, a proxy signcrypter with identity $I_{p}$, and a receiver with identity $I_{b}$.

Setup is an initialization algorithm that takes a security parameter $k$ as input. The PKG runs this algorithm and outputs the master key $s$ along with a set of system parameters $\beta$.

Extract is an extraction algorithm that takes $\beta$ and an identity $I_{i}(i$ is $a, p, b)$ of some user as input. The PKG runs this algorithm and outputs the public key $Y_{i}$ and private key $S_{i}$ of this user. Keep in mind that $I_{a}$ is the identity of the original signcrypter, $I_{p}$ is the identity of the proxy signcrypter, and $I_{b}$ is the identity of the receiver.

Apparently, the public and private key pair of the original signcrypter, proxy signcrypter, and receiver is $\left\langle Y_{a}, S_{a}\right\rangle$, $<$ $Y_{p}, S_{p}>$, and $<Y_{b}, S_{b}>$, respectively.
PKeyGen is a proxy key generation algorithm that takes $<\beta, I_{a}, I_{p}>$ as input. The original signcrypter with identity $I_{a}$ generates an authorization certificate $m_{w}$ and then interacts with the proxy signcrypter with identity $I_{p}$. Finally, this algorithm outputs a proxy signcryption key $S_{a p}$ for the proxy signcrypter.

ProxyS is a proxy signcryption algorithm that takes < $\beta, m, m_{w}, I_{a}, I_{b}, I_{p}, Y_{p}, Y_{b}, S_{a p}>$ as input. The proxy signcrypter with identity $I_{p}$ runs this algorithm and outputs a ciphertext $\sigma$ on message $m$ to the receiver with identity $I_{b}$.

Uns is an unsigncryption algorithm that takes $<\beta, \sigma, m_{w}, I_{a}, I_{b}, I_{p}, Y_{a}, Y_{p}, Y_{b}, S_{b}>$ as input. The receiver with identity $I_{b}$ runs this unsigncryption algorithm and outputs a plaintext $m$ or a symbol $\perp$.

3.2. Security Model. In this section, we describe the formal security model of the universally composable IBPSP. A universally composable IBPSP must satisfy the confidentiality (IND-CCA2 security) and unforgeability (UF-CMA security). Keep in mind that we do not allow the queries when the identities of the original signcrypter, proxy signcrypter, or receiver are the same.

For the confidentiality of the universally composable IBPSP, we adopt the IND-CCA2 security model. Now, we describe the interactive game IND-IBPS-CCA2 played between an adversary $\mathscr{A}$ and a challenger $C$.

IND-IBPS-CCA2: First of all, $C$ runs the initialization algorithm to obtain a set of system parameters $\beta$ and the master key $s$. Then $C$ keeps the secrecy of $s$ but returns $\beta$ to $\mathscr{A}$.

Phase 1. $A$ makes a sequence of a polynomial bounded number of queries in an adaptive fashion.

Extraction queries: $\mathscr{A}$ submits a query of identity $I_{i}$ to the extraction oracle. $C$ returns the private key $S_{i}$ and public key $Y_{i}$ by a call to the extraction algorithm.

Proxy key queries: $\mathscr{A}$ submits a query of the trituple < $I_{a}, I_{p}, m_{w}>$ to the proxy key generation oracle. $C$ returns the proxy key $S_{a p}$ by a call to the proxy key generation algorithm.

Proxy signcryption queries: $\mathscr{A}$ submits a query of the quaternion $<I_{a}, I_{b}, I_{p}, m>$ to the proxy signcryption oracle. $C$ returns the ciphertext $\sigma$ on message $m$ by a call to the proxy signcryption algorithm.

Unsigncryption queries: $\mathscr{A}$ submits a query of the quaternion $<I_{a}, I_{b}, I_{p}, \sigma>$ to the unsigncryption oracle. $C$ returns a plaintext $m$ or a symbol $\perp$ by a call to the unsigncryption algorithm.

Challenge. At the end of Phase 1, the adversary outputs two messages $<m_{0}, m_{1}>\in\{0,1\}^{l}$ and quaternion < $I_{a}{ }^{*}, I_{b}{ }^{*}, I_{p}{ }^{*}, m_{w}{ }^{*}>$ on which it wishes to be challenged. In Phase 1, the adversary cannot query the private key of identity $I_{b}{ }^{*}$. $C$ selects a random $\delta \in\{0,1\}$ and obtains a challenge ciphertext $\sigma^{*}$ on message $m_{\delta}$ by a call to the proxy signcryption algorithm. Finally, $C$ returns $\sigma^{*}$ as the answer.

Phase 2. $\mathscr{A}$ makes another sequence of queries in an adaptive fashion as in the first phase. $C$ answers these queries as in the 
first phase. However, the adversary cannot extract the private key of identity $I_{b}{ }^{*}$ and cannot make the unsigncryption query on $\sigma^{*}$.

At the end of the game, the adversary outputs a guess $\delta^{\prime} \in\{0,1\}$. If $\delta=\delta^{\prime}$, this shows that the adversary wins INDIBPS-CCA 2 above.

Definition 4 (confidentiality). A universally composable IBPSP is said to have the IND-CCA2 security if no probabilistic polynomial time adversary $\mathscr{A}$ wins IND-IBPS-CCA2 with nonnegligible advantage.

For the unforgeability of the universally composable IBPPSP, we adopt the UF-CMA security model. Keep in mind that, in the security models, $\mathscr{A}_{p}$ is in possession of the private key of the proxy signcrypter and $\mathscr{A}_{o}$ owns the private key of the original signcrypter.

UF-IBPS-CMA-I: This is an interactive game performed between an adversary $\mathscr{A}_{p}$ and a challenger $C$.

First of all, $C$ generates a set of system parameters $\beta$ and the master key $s$ by a call to $\operatorname{Setup}\left(1^{k}\right)$. It then keeps the secrecy of $s$ but delivers $\beta$ to the adversary.

Queries. $\mathscr{A}_{p}$ makes a series of a polynomial bounded number of queries in an adaptive manner as in the first phase in IND-IBPS-CCA2. Moreover, $C$ answers these queries as in the first phase in IND-IBPS-CCA2.

Forgery. At the end of queries, $\mathscr{A}_{p}$ outputs a forged ciphertext $<I_{a}{ }^{*}, I_{b}{ }^{*}, I_{p}{ }^{*}, \sigma^{*}>$ to $C$. In queries, $\mathscr{A}_{p}$ cannot issue the private key query of identity $I_{p}{ }^{*}$. If the unsigncryption verification equality holds, this indicates that $\mathscr{A}_{p}$ wins UF-IBPS-CMA-I.

UF-IBPS-CMA-II: This is an interactive game performed between an adversary $\mathscr{A}_{o}$ and a challenger $C$.

First of all, $C$ obtains the master key $s$ and a set of system parameters $\beta$ by a call to $\operatorname{Setup}\left(1^{k}\right)$. It then returns $\beta$ to the adversary but keeps the secrecy of $s$.

Queries. $\mathscr{A}_{o}$ adaptively makes a series of a polynomial bounded number of queries as in Phase 1 in IND-IBPSCCA2. $C$ answers these queries as in Phase 1 in IND-IBPSCCA2.

Forgery. At the end of queries, $\mathscr{A}_{o}$ outputs a forged ciphertext $<I_{a}{ }^{*}, I_{b}{ }^{*}, I_{p}{ }^{*}, \sigma^{*}>$ to $C$. In queries, $\mathscr{A}_{o}$ cannot extract the private key of identity $I_{a}{ }^{*}$. If the verification equality holds, this shows that $\mathscr{A}_{0}$ wins UF-IBPSCMA-II.

Definition 5 (unforgeability). A universally composable IBPSP is said to have UF-CMA-I security if no probabilistic polynomial time adversary $\mathscr{A}_{p}$ wins UF-IBPS-CMA-I with nonnegligible advantage. In a similar manner, a universally composable IBPSP is said to have the UF-CMA-II security if probabilistic polynomial time adversary $\mathscr{A}_{o}$ wins UF-IBPSCMA-II with nonnegligible advantage. Hence, a universally composable IBPSP is UF-CMA secure if it is both UF-CMA-I and UF-CMA-II secure.

\section{Concrete Protocol}

In this section, we devise a concrete example of the universally composable IBPSP; its algorithm details are described as follows.

4.1. Setup. In this system initialization algorithm with the input $1^{k}$, the PKG obtains the master key $s$ and a set of system parameters $\beta$ by carrying out the steps as below.

Let $G_{1}$ denote a cyclic addition group with prime order $q$ and let $G_{2}$ denote a cyclic multiplication group with the same prime order. Moreover, $P$ is a generator of addition group $G_{1}$ and $e: G_{1} \times G_{1} \longrightarrow G_{2}$ is a bilinear map. The PKG chooses four cryptography hash functions:

$$
\begin{array}{r}
h_{1}:\{0,1\}^{*} \longrightarrow G_{1} \\
h_{2}:\{0,1\}^{t} \times G_{1} \longrightarrow Z_{q}^{*} \\
h_{3}:\{0,1\}^{t} \times G_{1} \times G_{2} \longrightarrow\{0,1\}^{l} \\
h_{4}:\{0,1\}^{l} \times\{0,1\}^{t 3} \times G_{1} \times G_{2} \longrightarrow Z_{q}^{*}
\end{array}
$$

Then the PKG selects a random $s \in Z_{q}^{*}$ as the system master key and calculates the system public key $y=s P$. Finally, the PKG keeps the secrecy of master key $s$ but publishes a set of the system parameters

$$
\beta=\left\langle G_{1}, G_{2}, q, e, t, l, P, y, h_{1} \sim h_{4}\right\rangle .
$$

4.2. Extract. In this extraction algorithm with the input $\beta$ and an identity $I_{i}(i$ is $a, b, p)$ of some user, the PKG carries out the following calculations to achieve this user's public key and private key. Concretely speaking, the PKG calculates the public and private key for the original signcrypter with identity $I_{a}$ as follows:

$$
\begin{aligned}
& Y_{a}=h_{1}\left(I_{a}\right), \\
& S_{a}=s Y_{a} .
\end{aligned}
$$

In the same manner, the PKG calculates the public and private key for the proxy signcrypter with identity $I_{p}$ as follows:

$$
\begin{aligned}
& Y_{p}=h_{1}\left(I_{p}\right), \\
& S_{p}=s Y_{p} .
\end{aligned}
$$

We also readily obtain the public and private key for the receiver with identity $I_{b}$ as follows:

$$
\begin{aligned}
& Y_{b}=h_{1}\left(I_{b}\right), \\
& S_{b}=s Y_{b} .
\end{aligned}
$$

4.3. PKeyGen. In this proxy key generation algorithm with the input $\left\langle\beta, I_{a}, I_{p}>\right.$, the original signcrypter with identity $I_{a}$ generates an authorization certificate $m_{w}$ which includes the identity information of the original signcrypter and proxy 
signcrypter together with other restriction contents. The original signcrypter selects a random $k_{a} \in Z_{q}{ }^{*}$ and calculates

$$
\begin{aligned}
U_{a} & =k_{a} Y_{a} \\
V & =h_{2}\left(m_{w}, U_{a}\right) S_{a} .
\end{aligned}
$$

After that, the original signcrypter delivers the trituple $<U_{a}, V, m_{w}>$ to the proxy signcrypter with identity $I_{p}$. Upon receiving $\left\langle U_{a}, V, m_{w}\right\rangle$, the proxy signcrypter verifies whether the equality holds as below:

$$
e(P, V)=e\left(y, Y_{a}\right)^{h_{2}\left(m_{w}, U_{a}\right)} .
$$

If the verification is true, the proxy signcrypter calculates the proxy signcryption key

$$
S_{a p}=S_{p}+V
$$

4.4. ProxyS. In this proxy signcryption algorithm with the input $<\beta, m, m_{w}, I_{a}, I_{p}, I_{b}, Y_{p}, Y_{b}, S_{a p}>$, the proxy signcrypter with identity $I_{p}$ obtains a ciphertext $\sigma$ on message $m$ and delivers it to the receiver with identity $I_{b}$.

Concretely speaking, the proxy signcrypter selects a random $k_{p} \in Z_{q}^{*}$ to set $U_{p}=k_{p} P$ and then continues to calculate

$$
\begin{aligned}
& R=e\left(y, Y_{b}\right)^{k_{p}} \\
& c=m \oplus h_{3}\left(m_{w}, U_{p}, R\right) \\
& \mu=h_{4}\left(m, I_{a}, I_{p}, I_{b}, U_{p}, R\right) \\
& S=\mu S_{a p}+k_{p} Y_{p} .
\end{aligned}
$$

Finally, the ciphertext $\sigma=<c, m_{w}, S, U_{a}, U_{p}>$ is sent to the receiver.

4.5. Uns. In this unsigncryption algorithm with the input < $\beta, \sigma, m_{w}, I_{a}, I_{b}, I_{p}, Y_{a}, Y_{p}, Y_{b}, S_{b}>$, the receiver with identity $I_{b}$ recovers $m$ using two equalities as follows:

$$
\begin{aligned}
& R=e\left(U_{p}, S_{b}\right) \\
& m=c \oplus h_{3}\left(m_{w}, U_{p}, R\right) .
\end{aligned}
$$

After that, the receiver continues to calculate

$$
\mu=h_{4}\left(m, I_{a}, I_{p}, I_{b}, U_{p}, R\right)
$$

Finally, this receiver checks whether the verification equality holds as follows:

$$
e(P, S)=e\left(y, h_{2}\left(m_{w}, U_{a}\right) Y_{a}+Y_{p}\right)^{\mu} e\left(U_{p}, Y_{p}\right)
$$

If the above verification is true, this illustrates that $\sigma$ is valid; otherwise, it is invalid.
It is easy for us to verify the correctness of our concrete protocol instance using three equalities as follows:

$$
\begin{aligned}
e(P, V) & =e\left(P, h_{2}\left(m_{w}, U_{a}\right) S_{a}\right)=e\left(P, s Y_{a}\right)^{h_{2}\left(m_{w}, U_{a}\right)} \\
& =e\left(y, Y_{a}\right)^{h_{2}\left(m_{w}, U_{a}\right)} \\
R & =e\left(y, Y_{b}\right)^{k_{p}}=e\left(U_{p}, s Y_{b}\right)=e\left(U_{p}, S_{b}\right) \\
e(P, S) & =e\left(P, \mu S_{a p}+k_{p} Y_{p}\right) \\
& =e\left(P, S_{p}+V\right)^{\mu} e\left(P, k_{p} Y_{p}\right) \\
& =e\left(P, s Y_{p}+h_{2}\left(m_{w}, U_{a}\right) S_{a}\right)^{\mu} e\left(U_{p}, Y_{p}\right) \\
& =e\left(y, h_{2}\left(m_{w}, U_{a}\right) Y_{a}+Y_{p}\right)^{\mu} e\left(U_{p}, Y_{p}\right) .
\end{aligned}
$$

\section{Analysis of Semantic Security}

In this section, we describe the proof process of the confidentiality and unforgeability of our protocol instance in the random oracle model.

\subsection{Confidentiality}

Theorem 6. If no probabilistic polynomial time adversary $\mathscr{A}$ wins IND-IBPS-CCA2 in Definition 4 with nonnegligible advantage $\varepsilon$ by asking $l_{i}$ queries to the $h_{i}(i=1,2,3,4)$ oracle, $l_{e}$ queries to the extraction oracle, and $l_{a p}$ queries to the proxy key generation oracle, then there exists an algorithm $C$ that can solve the gap $B D H$ problem with the advantage $\varepsilon^{\prime}$ ( $e$ is the base of natural logarithm), where

$$
\varepsilon^{\prime} \geq \frac{\varepsilon}{e l_{3}\left(l_{e}+l_{a p}\right)} .
$$

Proof. Assume the algorithm $C$ obtains a random instance $<$ $P, a P, b P, c P>$ of the gap $\mathrm{BDH}$ problem, and the purpose of $C$ is to determine $R=(P, P)^{a b c} \in G_{2}$. For this purpose, $C$ runs the adversary $\mathscr{A}$ as a subroutine and acts as the role of its challenger in the following interactive game.

At the start of the game, $C$ runs the system initialization algorithm with the security parameter $k$ and obtains a set of system parameters $\beta$ with $y=a P$. Then $C$ delivers $\beta$ to the adversary. In the whole game, $C$ maintains five lists $L_{1} \sim L_{4}$ and $L_{a p}$ which are empty in the beginning; $L_{1} \sim L_{4}$ are used to trace the $h_{i}(i=1,2,3,4)$ oracle and $L_{a p}$ is used to trace the extraction oracle and proxy key generation oracle.

Phase 1. A submits a series of a polynomial bounded number of queries in an adaptive fashion.

$h_{1}$ queries: $C$ first selects an identity $I_{\lambda}\left(1 \leq \lambda \leq l_{1}\right)$ as the target identity in the challenge phase. Bear in mind that $l_{1}$ is the query time to the $h_{1}$ oracle. Let $\gamma$ be the probability of $I_{i}=I_{\lambda}$, and the value of $\gamma$ will be determined later. $\mathscr{A}$ submits a query of identity $I_{i}$ to the $h_{1}$ oracle. If there is a matching tuple in the list $L_{1}, C$ returns the public key $Y_{i}$ as the answer; otherwise, $C$ considers two cases as the response to this query. 
Case 1. If it is the $\lambda$ th query, $C$ sets $Y_{i}=h_{1}\left(I_{i}\right)=b P$. It then delivers public key $Y_{i}$ to the adversary and records $<I_{i}, Y_{i},-,->$ to the list $L_{1}$.

Case 2. If it is not the $\lambda$ th query, $C$ uses a random $x_{i} \in Z_{q}^{*}$ of its choice to calculate $Y_{i}=h_{1}\left(I_{i}\right)=x_{i} P$. It then returns $Y_{i}$ as the answer and records $<I_{i}, Y_{i}, x_{i},->$ to the list $L_{1}$.

$h_{2}$ queries: $\mathscr{A}$ submits a query to the $h_{2}$ oracle. If there is a matching tuple in the list $L_{2}, C$ returns $f$ as the answer; otherwise, $C$ returns a random $f \in Z_{q}^{*}$ of its choice and records $<m_{w}, U_{a}, f>$ to the list $L_{2}$.

$h_{3}$ queries: $\mathscr{A}$ submits a query to the $h_{3}$ oracle. If there exists a matching tuple in the list $L_{3}, C$ returns $\phi$ as the answer; otherwise, $C$ returns a random $\phi \in\{0,1\}^{l}$ of its choice and records $<m_{w}, R, U_{p}, \phi>$ to the list $L_{3}$.

$h_{4}$ queries: $\mathscr{A}$ submits a query to the $h_{4}$ oracle. If there is a matching tuple in the list $L_{4}, C$ delivers $\mu$ to the adversary; otherwise, $C$ returns a random $\mu \in Z_{q}^{*}$ of its choice and stores $<m, I_{a}, I_{p}, I_{b}, U_{p}, R, \mu>$ to the list $L_{4}$.

Extraction queries: $\mathscr{A}$ submits a query of identity $I_{i}$ to the extraction oracle. $C$ terminates this simulation if $I_{i}=I_{\lambda}$; otherwise, $C$ calculates $S_{i}=x_{i} a P=x_{i} y$ and then returns $<Y_{i}, S_{i}>$ and stores $<I_{i}, Y_{i}, x_{i}, S_{i}>$ into the list $L_{1}$.

Proxy key queries: $\mathscr{A}$ submits a proxy key query of the trituple $<I_{a}, I_{p}, m_{w}>$. Let us assume the adversary has made the $h_{1}$ and $h_{2}$ oracle queries along with key extraction queries before the proxy key query. If $I_{a}=I_{\lambda}, C$ terminates this simulation; otherwise, $C$ makes use of a random $k_{a} \in Z_{q}^{*}$ of its choice to calculate

$$
\begin{aligned}
U_{a} & =k_{a} Y_{a} \\
V & =f x_{a} y .
\end{aligned}
$$

If the verification equality $e(P, V)=e\left(y, Y_{a}\right)^{f}$ holds, $C$ calculates the proxy key $S_{a p}$ using the equality as below

$$
S_{a p}=x_{p} y+V
$$

and delivers $S_{a p}$ to the adversary. Eventually, $C$ stores < $m_{w}, S_{a p}, U_{a}, V>$ into the list $L_{a p}$.

Proxy signcryption queries: Assume the adversary has made various hash oracle queries together with the extraction and proxy key generation query before a proxy signcryption query. $\mathscr{A}$ requests a query of $<I_{a}, I_{b}, I_{p}, m_{w}, m>$ to the proxy signcryption oracle. $C$ considers two cases as the response to this proxy signcryption query.

Case 1. If $I_{p} \neq I_{\lambda}, C$ returns obtains a ciphertext $\sigma$ by a call to the actual proxy signcryption algorithm and delivers this ciphertext to the adversary.

Case 2. If $I_{p}=I_{\lambda}, C$ selects two randoms $v, k_{p} \in Z_{q}^{*}$ and calculates

$$
\begin{gathered}
U_{p}=k_{p} P-v y \\
R=e\left(U_{p}, S_{b}\right) .
\end{gathered}
$$

$C$ continues to calculate $c=m \oplus \phi$ and stores < $m_{w}, U_{p}, R, \phi>$ into the list $L_{3}$. Then $C$ sets $\mu=v$ and adds $<m, I_{a}, I_{p}, I_{b}, U_{p}, R, \mu>$ into the list $L_{4}$. Finally, $C$ calculates

$$
S=\mu f x_{a} y+k_{p} Y_{p}
$$

and returns $\sigma=<c, m_{w}, S, U_{a}, U_{p}>$ as the answer.

It is very effortless for the adversary to verify the validity of the ciphertext $\sigma$ using the equality as below.

$$
\begin{aligned}
& e\left(y, h_{2}\left(m_{w}, U_{a}\right) Y_{a}+Y_{p}\right)^{\mu} e\left(U_{p}, Y_{p}\right) \\
& =e\left(y, f Y_{a}+Y_{p}\right)^{\mu} e\left(k_{p} P-v y, Y_{p}\right) \\
& =e\left(y, \mu f x_{a} P\right) e\left(y, v Y_{p}\right) e\left(P, k_{p} Y_{p}\right) e\left(y, Y_{p}\right)^{-v} \\
& =e\left(P, \mu f x_{a} y\right) e\left(P, k_{p} Y_{p}\right)=e\left(P, \mu f x_{a} y+k_{p} Y_{p}\right) \\
& =e(P, S) .
\end{aligned}
$$

Unsigncryption queries: Assume $\mathscr{A}$ has queried various hash oracles together with the extraction oracle and proxy key generation oracle before an unsigncryption query. $\mathscr{A}$ requests an unsigncryption query of the quaternion < $I_{a}, I_{b}, I_{p}, m_{w}, \sigma>$. $C$ considers two cases as the response to this unsigncryption query.

Case 1. If $I_{b} \neq I_{\lambda}, C$ returns a result by a call to the actual unsigncryption algorithm.

Case 2. If $I_{b}=I_{\lambda}, C$ goes over the list $L_{3}$ to seek the tuple $<m_{w}, U_{p}, R, \phi>$ for different $R$ such that $\mathcal{O}_{D B D H}$ returns 1 when the adversary made a query on $<y, Y_{b}, U_{b}, R>$. If there exists this case, $C$ calculates

$$
\begin{aligned}
& m=c \oplus \phi \\
& \mu=h_{4}\left(m, I_{a}, I_{p}, I_{b}, U_{p}, R\right) .
\end{aligned}
$$

Finally, $C$ checks whether the verification equality holds as below:

$$
e(P, S)=e\left(y, h_{2}\left(m_{w}, U_{a}\right) Y_{a}+Y_{p}\right)^{\mu} e\left(U_{p}, Y_{p}\right) .
$$

If the verification is true, $C$ returns $m$; otherwise, $C$ returns $\perp$.

Challenge. As the adversary decides to end the first phase, it outputs $m_{0}$ and $m_{1}$ with the same length along with the quaternion $<I_{a}{ }^{*}, I_{b}{ }^{*}, I_{p}{ }^{*}, m_{w}{ }^{*}>$ on which it wishes to be challenged. Assume the adversary has queried various hash oracles together with the extraction and proxy key generation oracle before a challenge query. In the first phase, the private key of identity $I_{b}{ }^{*}$ cannot be extracted. Upon receiving a challenge query, $C$ deals with this query by considering two cases as follows.

Case 1. If $I_{p}{ }^{*}=I_{\lambda}, C$ terminates this simulation. 
Case 2. If $I_{p}{ }^{*} \neq I_{\lambda}, C$ selects a random $\delta \in\{0,1\}$ and $R^{*} \in G_{2}$. Then $C$ sets $U_{p}{ }^{*}=c P$ and calculates

$$
\begin{aligned}
c^{*} & =m_{\delta} \oplus \phi^{*} \\
\mu^{*} & =h_{4}\left(m_{\delta}, I_{a}^{*}, I_{p}{ }^{*}, I_{b}{ }^{*}, U_{p}{ }^{*}, R^{*}\right) .
\end{aligned}
$$

$C$ stores $<m_{w}{ }^{*}, U_{p}{ }^{*}, R^{*}, \phi^{*}>$ into the list $\mathrm{L}_{3}$ and records $<m_{\delta}, I_{a}{ }^{*}, I_{p}{ }^{*}, I_{b}{ }^{*}, U_{p}{ }^{*}, R^{*}, \mu^{*}>$ to the list $\mathrm{L}_{4}$. Finally, $\mathrm{C}$ continues to calculate

$$
S^{*}=\mu^{*} S_{a p}{ }^{*}+x_{p}^{*} U_{p}^{*}
$$

and returns $\sigma^{*}=<c^{*}, m_{w}{ }^{*}, S^{*}, U_{a}{ }^{*}, U_{p}{ }^{*}>$ as challenge ciphertext.

Phase 2. $\mathscr{A}$ issues another sequence of oracle queries in an adaptive fashion as the first phase. In addition, $C$ answers these oracle queries as in the first phase. However, $\mathscr{A}$ cannot extract the private key of identity $I_{b}{ }^{*}$ and cannot make use of $\sigma^{*}$ to query the unsigncryption oracle.

At the end of the game, the adversary outputs a guess $\delta^{\prime} \epsilon$ $\{0,1\}$ of $\delta$. If $\delta=\delta^{\prime}, C$ outputs the solution of the gap BDH problem instance:

$$
R^{*}=e\left(U_{p}{ }^{*}, S_{b}{ }^{*}\right)=e\left(c P, a Y_{b}{ }^{*}\right)=e(P, P)^{a b c}
$$

Analysis of probability. In the following, we analyze the success probability of $C$ in solving the gap BDH problem.

According to the detailed description in the interactive game, we have that the probability of $C$ not terminating the simulation in the first phase or second phase is $\gamma^{l_{e}+l_{a p}}$. And the probability of $C$ not terminating the simulation in the challenge phase is $1-\gamma$. Thus, the probability of $C$ not terminating the simulation is $\gamma^{l_{e}+l_{a p}}(1-\gamma)$ whose value is maximized at

$$
\gamma=1-\frac{1}{1+l_{e}+l_{a p}} .
$$

Referring to the method of the probability analysis in [20], we obtain that the probability of $C$ not terminating the simulation is at least

$$
\frac{1}{e\left(l_{e}+l_{a p}\right)} \text {. }
$$

At the same time, the probability of the adversary querying the $h_{3}$ oracle is $1 / l_{3}$. Hence, the probability of $C$ in solving the gap $\mathrm{BDH}$ problem is at least

$$
\frac{\varepsilon}{e l_{3}\left(l_{e}+l_{a p}\right)} \text {. }
$$

If the adversary succeeds with probability $\varepsilon, C$ can solve the gap $\mathrm{BDH}$ problem with probability $\varepsilon^{\prime}$. It contracts with the initial gap $\mathrm{BDH}$ assumption. Therefore, $\varepsilon$ must be negligible if the gap $\mathrm{BDH}$ problem is hard.

\subsection{Unforgeability}

Theorem 7. If no probabilistic polynomial time adversary $\mathscr{A}_{p}$ (or $\left.\mathscr{A}_{o}\right)$ wins UF-IBPS-CMA-I (or UF-IBPS-CMA-II) with nonnegligible advantage $\varepsilon$ by making $l_{i}$ queries to the $h_{i}$ $(i=1,2,3,4)$ oracle, $l_{e}$ queries to the extraction oracle, $l_{a p}$ queries to the proxy key generation oracle, and $l_{\text {usc }}$ queries to the unsigncryption oracle, then there exist two cases. (1) In UFIBPS-CMA-I, there exists an algorithm $C$ which can solve the $\mathrm{CDH}$ problem with the advantage $\varepsilon_{1}$, where

$$
\varepsilon_{1} \geq \frac{\varepsilon}{e l_{u s c}\left(l_{e}+l_{a p}\right)} .
$$

(2) In UF-IBPS-CMA-II, there is an algorithm $C$ which can solve the $\mathrm{CDH}$ problem with the advantage $\varepsilon_{2}$, where

$$
\varepsilon_{2} \geq \frac{\varepsilon}{e l_{e} l_{u s c}}
$$

Proof. Give a random instance $\langle P, a P, b P>$ of the $\mathrm{CDH}$ problem, and the aim of $C$ is to determine the value of $a b P \in$ $G_{1}$. In order to achieve this aim, the algorithm $C$ runs the adversary $\mathscr{A}_{p}$ or $\mathscr{A}_{o}$ as a subroutine and acts as the role of the challenger of $\mathscr{A}_{p}$ or $\mathscr{A}_{o}$ in the interactive game.

Initial. $C$ obtains a set of system parameters $\beta$ with $y=$ $a P$ by a call to the initialization algorithm and delivers $\beta$ to $\mathscr{A}_{p}$ or $\mathscr{A}_{o}$. In the interactive game, $C$ needs to maintain five lists $L_{1} \sim L_{4}$ and $L_{a p}$ which are empty in the beginning, and these lists make use of tracing the relevant random oracles.

Queries. These are the same as those in Phase 1 in Theorem 6.

Forgery. At the end of these queries, the response to the forgery is described as follows.

(1) As the queries are over, $\mathscr{A}_{p}$ outputs a forged ciphertext $\sigma^{*}=<c^{*}, m_{w}{ }^{*}, S^{*}, U_{a}{ }^{*}, U_{p}{ }^{*}>$ to $C$. In queries, $\mathscr{A}_{p}$ cannot extract the private key of identity $I_{p}{ }^{*}$ and $\sigma^{*}$ should not be the response of any proxy signcryption oracle query made by $\mathscr{A}_{p}$. $C$ considers two cases as the response to this query

Case 1. If $I_{p}{ }^{*}=I_{\lambda}$ and $I_{a}{ }^{*}=I_{\lambda}, C$ terminates this simulation.

Case 2. If $I_{p}{ }^{*} \neq I_{\lambda}$ and $I_{a}{ }^{*}=I_{\lambda}, C$ queries various hash oracles to obtain $<x_{p}{ }^{*}, f^{*}, \mu^{*}>$ and $Y_{a}{ }^{*}=b P$ from the list $L_{1}$. Then $C$ outputs the solution of the $\mathrm{CDH}$ problem instance

$$
a b P=\frac{S^{*}-\mu^{*} S_{p}{ }^{*}-x_{p}{ }^{*} U_{p}{ }^{*}}{\mu^{*} f^{*}} .
$$

If $C$ wins in the interactive game above, this shows that the verification equality must hold:

$$
\begin{aligned}
& e\left(P, S^{*}\right) \\
& \quad=e\left(y, h_{2}\left(m_{w}{ }^{*}, U_{a}{ }^{*}\right) Y_{a}{ }^{*}+Y_{p}{ }^{*}\right)^{\mu^{*}} e\left(U_{p}{ }^{*}, Y_{p}{ }^{*}\right) \\
&=e\left(y, \mu^{*} f^{*} Y_{a}{ }^{*}\right) e\left(y, \mu^{*} Y_{p}{ }^{*}\right) e\left(P, x_{p}{ }^{*} U_{p}{ }^{*}\right) \\
&=e\left(P, \mu^{*} f^{*} a b P\right) e\left(P, \mu^{*} S_{p}{ }^{*}\right) e\left(P, x_{p}{ }^{*} U_{p}{ }^{*}\right) .
\end{aligned}
$$


Analysis of probability. Now, we analyze the success probability that $C$ obtains the solution of the $\mathrm{CDH}$ problem instance.

From the interactive game, we know that the probability of $C$ not aborting the game in the first phase or second phase is $\gamma^{l_{e}+l_{a p}}$. And the probability of $C$ not aborting the execution of game in the challenge phase is $1-\gamma$. Thus, the probability of $C$ not aborting the execution of game is $\gamma^{l_{e}+l_{a p}}(1-\gamma)$. Referring to the probability analysis in Theorem 6, we can obtain that the probability of $C$ not aborting the execution of game is at least

$$
\frac{1}{e\left(l_{e}+l_{a p}\right)}
$$

Furthermore, the probability that $\sigma^{*}$ passes the unsigncryption verification is $1 / l_{u s c}$. Therefore, the probability of $C$ in solving the $\mathrm{CDH}$ problem is at least

$$
\frac{\varepsilon}{e l_{u s c}\left(l_{e}+l_{a p}\right)}
$$

(2) At the end of the queries, $\mathscr{A}_{o}$ outputs a forged ciphertext $\sigma^{*}=<c^{*}, m_{w}{ }^{*}, S^{*}, U_{a}{ }^{*}, U_{p}{ }^{*}>$ to $C$, where $U_{p}{ }^{*}=$ $y$. In queries, $\mathscr{A}_{o}$ cannot issue the private key query of identity $I_{a}{ }^{*}$ and $\sigma^{*}$ should not be the response of any proxy signcryption oracle query made by $\mathscr{A}_{o}$. It is needful for $C$ to deal with this query by considering two cases as below

Case 1. If $I_{a}{ }^{*}=I_{\lambda}$ and $I_{p}{ }^{*}=I_{\lambda}, C$ terminates this simulation.

Case 2. If $I_{a}{ }^{*} \neq I_{\lambda}$ and $I_{p}{ }^{*}=I_{\lambda}, C$ calls various hash oracles to achieve $<x_{p}{ }^{*}, f^{*}, \mu^{*}>$ and $Y_{p}{ }^{*}=b P$ from the list $L_{1}$. Then $C$ outputs the solution of the $\mathrm{CDH}$ problem instance:

$$
a b P=\frac{S^{*}-\mu^{*} f^{*} x_{p}^{*} y}{\mu^{*}+1} .
$$

If $C$ wins in the interactive game above, this indicates that the verification equality must hold:

$$
\begin{aligned}
e\left(P, S^{*}\right) \\
\quad=e\left(y, h_{2}\left(m_{w}{ }^{*}, U_{a}{ }^{*}\right) Y_{a}{ }^{*}+Y_{p}{ }^{*}\right)^{\mu^{*}} e\left(U_{p}{ }^{*}, Y_{p}{ }^{*}\right) \\
\quad=e\left(y, \mu^{*} f^{*} Y_{a}{ }^{*}\right) e\left(y, \mu^{*} Y_{p}{ }^{*}\right) e(y, b P) \\
=e\left(P, \mu^{*} f^{*} x_{a}{ }^{*} y\right) e\left(P, \mu^{*} a b P\right) e(P, a b P) .
\end{aligned}
$$

Analysis of probability. Now, Let us analyze the success probability of $C$ in solving the $\mathrm{CDH}$ problem.

From the interactive game above, we obtain that the probability of $C$ not aborting the game in the first phase or second phase is $\gamma^{l_{e}}$ and the probability of $C$ not aborting the game in the challenge phase is $1-\gamma$. Thus, the probability of $C$ not aborting the game is $\gamma^{l_{e}}(1-\gamma)$. Referring to the probability analysis in Theorem 6, we have that the probability of $C$ not aborting the game is at least $1 / e l_{e}$.
In addition, the probability that $\sigma^{*}$ passes the unsigncryption verification is $1 / l_{u s c}$. Therefore, the probability of $C$ in solving the $\mathrm{CDH}$ problem is at least

$$
\frac{\varepsilon}{e l_{e} l_{u s c}}
$$

If $\mathscr{A}_{1}$ or $\mathscr{A}_{0}$ succeeds with probability $\varepsilon, C$ can solve the $\mathrm{CDH}$ problem with probability $\varepsilon_{1}$ or $\varepsilon_{2}$. It contracts with the initial $\mathrm{CDH}$ assumption. Hence, $\varepsilon$ must be negligible if the $\mathrm{CDH}$ problem is hard.

\section{Analysis of UC Security}

6.1. Protocol $\pi_{\text {IBPSP. }}$. A concrete $\pi_{\text {IBPSP }}$ usually makes use of the PKG to control two algorithms: Setup and Extract. $\pi_{\text {IBPSP }}$ in the UC framework proceeds as below, with parties $P_{1}, \ldots, P_{n}$ and an adversary $\mathscr{A}$.

(1) Upon input $<$ PKG, Setup, sid $>$ from $\mathscr{A}$, the PKG verifies whether sid $=<\mathrm{PKG}, \operatorname{sid}^{\prime}>$. If not, the PKG ignores this request; else, the $\mathrm{PKG}$ obtains $\langle s, \beta>$ by a call to $\operatorname{Setup}\left(\mathbf{1}^{k}\right)$ and returns $\beta$

(2) Upon input < Extract, sid > from $\mathscr{A}, P_{i}$ obtains < $Y_{i}, S_{i}>$ by a call to $\operatorname{Extract}\left(\beta, s, I_{i}\right)$ and returns $<Y_{i}, S_{i}>$

(3) Upon input $<$ PKeyGen, sid $>$ from $\mathscr{A}, P_{i}$ obtains $S_{a p}$ by a call to $\operatorname{PKeyGen}\left(\beta, I_{a}, I_{p}\right)$ and returns $S_{a p}$

(4) Upon input < ProxyS, sid, $m>$ from $\mathscr{A}, P_{i}$ obtains a ciphertext $\sigma$ by a call to the algorithm $\operatorname{ProxyS}(\beta$, $\left.m, m_{w}, I_{a}, I_{b}, I_{p}, Y_{b}, Y_{p}, S_{a p}\right)$ and returns $\sigma$

(5) Upon input < Usc, sid, $\sigma>$ from $\mathscr{A}, P_{i}$ obtains $m \longleftarrow \operatorname{Uns}\left(\beta, \sigma, m_{w}, I_{a}, I_{b}, I_{p}, Y_{a}, Y_{p}, S_{b}\right)$ and then returns $f \longleftarrow v(\beta, \sigma, m$, sid $)$

6.2. Ideal Functionality $\mathscr{F}_{\text {IBPSP. In this section, we introduce }}$ the definition of an ideal functionality $\mathscr{F}_{\text {IBPSP }}$ for the IBPSP, based on the security definitions in Section 2.2. $\mathscr{F}_{\text {IBPSP }}$ and $\pi_{\text {IBPSP }}$ have the exact same interface; that is to say, $\mathscr{F}_{\text {IBPSP }}$ can be realized by $\pi_{\text {IBPSP }}$ under certain condition. $\mathscr{F}_{\text {IBPSP }}$ proceeds as follows, with parties $P_{1}, \ldots, P_{n}$ and an ideal adversary $\mathcal{S} . v$ is a deterministic verification algorithm.

(1) Given < Setup, sid > from the PKG, verify whether sid $=<\mathrm{PKG}$, sid' $^{\prime}>$ for some sid'. If not, then ignore this request; else, forward $<$ Setup, sid $>$ to $\mathcal{S}$. Then, on receiving $<$ Setup, sid, $v>$ from $\mathcal{S}$, record $\langle$ sid, $v>$ and output $<\operatorname{sid}, v>$ to $\mathcal{S}$

(2) On receiving < Extract, sid $>$ from some party $P_{i}$, verify whether sid $=<P_{i}$, sid' $>$. If not, then ignore this request; else, forward $<$ Extract, sid $>$ to $\mathcal{S}$. Then, on receiving $<Y_{i}, S_{i}, v>$ from $\mathcal{S}$, record $<Y_{i}, S_{i}, v>$ and output $<Y_{i}, S_{i}>$ to $P_{i}$

(3) On receiving < PKeyGen, sid $>$ from $P_{p}$, verify whether sid $=<P_{p}$, sid' $>$. If not, then ignore this; else, forward $<$ PKeyGen, sid $>$ to $\mathcal{S}$. Then, on receiving $\left\langle S_{a p}, v\right\rangle$ from $\mathcal{S}$, record $\left\langle S_{a p}, v>\right.$ and output $S_{a p}$ to $P_{p}$

(4) On receiving $<$ ProxyS, sid, $v^{\prime}, m>$ for some $m$ from $P_{p}$, verify whether sid $=<P_{p}, \operatorname{sid}^{\prime}>$. If not, then ignore this. Else, do the following:

(1) If $P_{p}$ is not corrupt and $v=v^{\prime}$, deliver < ProxyS, sid, $|m|>$ to $\mathcal{S}$, where $|m|$ is the length of message 
$m$; else, deliver $<$ ProxyS, sid, $v^{\prime}, m>$ to $\delta$ (2) On receiving the replay $\sigma$ from $\mathcal{S}$, deliver $<$ ProxyS, sid, $v^{\prime}, m, \sigma>$ to the party $P_{b}$ and record $\langle m, \sigma\rangle$

(5) On input $\left\langle\right.$ Uns, sid, $\sigma>$ from $P_{b}$, verify whether sid $=<P_{b}, \operatorname{sid}^{\prime}>$. If not, then ignore this. Else, do the following:

(1) If $\langle m, \sigma\rangle$ has been recorded, then achieve $f \longleftarrow$ $v\left(\beta, \sigma, m\right.$, sid) and send $<m, f=1>$ to $P_{b}$ (2) Else, return $<$ Uns, sid, $\sigma>$ to $\mathcal{S}$ and obtain $m$ from $\mathcal{S}$, and then send $<m, f=0>$ to $P_{b}$

\subsection{Proof of UC Security}

Theorem 8. If $\pi_{I B P S P}$ satisfies the IND-CCA2 security, then $\pi_{I B P S P}$ can securely realize $\mathscr{F}_{\text {IBPSP }}$ in the UC framework.

Proof. Assume the protocol $\pi_{\text {IBPSP }}$ satisfies the IND-CCA2 security; then $\pi_{\text {IBPSP }}$ can securely realize $\mathscr{F}_{\text {IBPS }}$ in the UC framework. i.e., if no probabilistic polynomial time $\mathscr{A}$ wins IND-IBPS-CCA2 with nonnegligible advantage, then any environment $\mathscr{Z}$ cannot distinguish whether it is interacting with $\pi_{\text {IBPSP }}$ and $\mathscr{A}$ in the real-life model or with $\mathscr{F}_{\text {IBPSP }}$ and $\mathcal{S}$ in the ideal model.

Environment $\mathscr{Z}$ proceeds as follows.

(1) $\mathscr{Z}$ delivers a request on Setup. On receiving < Setup, sid $>$, $\mathscr{Z}$ inputs $\beta$ and activates $\mathscr{A}$

(2) On receiving a request on $<$ Extract, sid $>$ from $\mathscr{A}$, $\mathscr{Z}$ activates some party $P_{i}$ with input $<$ Extract, sid $>$ and outputs $<Y_{i}, S_{i}>$ to $\mathscr{A}$

(3) On receiving < PKeyGen, sid > from $\mathscr{A}, \mathscr{Z}$ activates $P_{a}$ and $P_{p}$ with $<$ PKeyGen, sid $>$ and outputs $S_{a p}$ to $\mathscr{A}$

(4) On receiving < ProxyS, sid, $m>$ from $\mathscr{A}$, $\mathscr{Z}$ activates $P_{p}$ with input $<$ ProxyS, sid, $m>$ and outputs $\sigma$ to $\mathscr{A}$

(5) On receiving a request on $\langle$ Uns, sid, $\sigma>$ from $\mathscr{A}, \mathscr{Z}$ activates $P_{b}$ with input $<$ Uns, sid, $\sigma>$ and outputs the value $f$ of $v(\sigma, m$, sid $)$

If $\mathscr{A}$ wins IND-IBPS-CCA2, $\mathscr{Z}$ outputs $f=1$. In addition, the probability that $\mathscr{Z}$ outputs 1 is 0 in the ideal model. Obviously, if $\pi_{\text {IBPSP }}$ satisfies the IND-CCA2 security, $\pi_{\text {IBPSP }}$ can securely realize $\mathscr{F}_{\text {IBPSP }}$ in the UC framework.

Theorem 9. If $\pi_{I B P S P}$ can securely realize $\mathscr{F}_{I B P S P}$ in the UC framework, then $\pi_{I B P S P}$ satisfies the IND-CCA2 security.

Proof. Here we construct an ideal adversary $\mathcal{S}$ such that no environment $\mathscr{Z}$ can tell with nonnegligible advantage whether it interacts with $\mathscr{F}_{\text {IBPSP }}$ and $\mathcal{S}$ in the ideal model or with $\pi_{\text {IBPSP }}$ and $\mathscr{A}$ in the real-life model.

$\mathcal{S}$ runs a copy of $\mathscr{A}$ and sends the input from $\mathscr{Z}$ to $\mathscr{A}$, and simultaneously any output from $\mathscr{A}$ is considered as the output of $\mathcal{S}$.

(1) On receiving < Setup, sid $>$ from $\mathscr{F}_{\text {IBPSP }}$, it runs the Setup algorithm and obtains $\langle\beta, s\rangle$, and then returns $<$ Setup, sid, $v, \beta>$

(2) On receiving $<$ Extract, sid $>$ from $\mathscr{F}_{\text {IBPSP }}$, it invokes the extraction algorithm to achieve $<Y_{i}, S_{i}>$ and then returns $<Y_{i}, S_{i}>$
(3) On receiving < PKeyGen, sid > from $\mathscr{F}_{\text {IBPSP }}$, it invokes the proxy key generation algorithm to obtain $S_{a p}$ and then returns $S_{a p}$

(4) On receiving < ProxyS, sid, $m>$ from $\mathscr{F}_{\text {IBPSP }}$, it runs the proxy signcryption algorithm to obtain $\sigma$ and then returns $\sigma$

(5) On receiving $<$ Uns, sid, $\sigma>$ from $\mathscr{F}_{\text {IBPSP }}$, it runs the unsigncryption algorithm to obtain $m$ and then returns $m$. Then it returns $f \longleftarrow v\left(\sigma, m\right.$, sid). If $P_{b}$ is corrupted, then deliver $<m, f=0>$ to all parties; else, deliver $<m, f=1>$ to $P_{b}$

In the following, we prove that the ideal adversary $\mathcal{S}$ simulates the real-life adversary $\mathscr{A}$.

(1) On receiving $\beta$ from the challenger $C, \mathscr{Z}$ is invoked. On receiving $<$ Setup, sid $>$ from $\mathscr{Z}$, it issues a request to $C$ that returns $\eta=(\operatorname{sid}, \beta)$ as the answer

(2) On receiving $<$ Extract, sid $>$ from $\mathscr{Z}$, it issues a request to $C$ that returns $<Y_{i}, S_{i}>$ as the answer

(3) On receiving $<$ PKeyGen, sid > from $\mathscr{Z}$, it issues a request to $C$ that returns $S_{a p}$ as the answer

(4) On receiving < ProxyS, sid, $m>$ from $\mathscr{Z}$, it issues a request to $C$ that responds as follows:

(1) If $P_{p}$ is uncorrupt, send < ProxyS, sid, $|m|>$ to $C$ that returns $\sigma^{\prime}$ as the answer

(2) If $P_{p}$ is corrupt, deliver $<$ ProxyS, sid, $m>$ to $C$ that returns $\sigma$ as the answer

(5) On input $\langle$ Uns, sid, $\sigma>$ from $\mathscr{Z}$, it issues a request to $C$ that responds as follows:

(1) If both $P_{p}$ and $P_{b}$ are not corrupt, then send < Uns, sid, $\sigma^{\prime}>$ to $C$ that returns $m^{\prime}$ as the answer

(2) If $P_{p}$ is corrupt and $P_{b}$ is not corrupt, then send < Uns, sid, $\sigma>$ to $C$ that returns $m^{\prime}$ as the answer

(3) If $P_{p}$ is not corrupt and $P_{b}$ is corrupt, then send < Uns, sid, $\sigma^{\prime}>$ to $C$ that returns $m^{\prime}$ as the answer

(4) If both $P_{p}$ and $P_{b}$ are corrupt, then deliver < Uns, sid, $\sigma>$ to $C$ that returns $m$ as the answer

Then, $\mathscr{Z}$ obtains $f \longleftarrow v(\beta, \sigma, m$, sid) and returns $f$. If $f=1$, this shows that the ciphertext is valid; else, it is invalid.

According to the running process, we define four events: for $\mathscr{Z}$, the occurrence of each event means that it cannot tell whether it interacts with $\pi_{\text {IBPSP }}$ and $\mathscr{A}$ in the real-life model or with $\mathscr{F}_{\text {IBPSP }}$ and $\mathcal{S}$ in the ideal model.

Event 1: $P_{p}$ and $P_{b}$ are both uncorrupt. In the reallife process, $P_{p}$ obtains $\sigma$ of $m$ by the proxy signcryption algorithm and sends $\sigma$ to $P_{b}$. And $P_{b}$ can unsigncrypt $\sigma$ to obtain $m$ and verifies the validity of $\sigma$. In the ideal process, $\mathcal{S}$ simulates $P_{b}$ to obtain $\sigma^{\prime}$ of $m^{\prime}$ of its choice and sends $\sigma^{\prime}$ to $P_{b} . \mathcal{S}$ answers with $m^{\prime}$ when $P_{b}$ unsigncrypts $\sigma^{\prime}$; the unsigncryption succeeds if $f=1$.

Event 2: $P_{p}$ is not corrupt and $P_{b}$ is corrupt. In the real-life process, $\mathscr{Z}$ cannot determine which message is signcrypted by $P_{p}$. In the ideal process, $\mathcal{S}$ simulates $P_{p}$ with $m^{\prime}$ of its choice to obtain $\sigma^{\prime}$ and forwards $\sigma^{\prime}$ to $\mathscr{A}$. It follows that $\mathscr{Z}$ cannot distinguish $\langle m, \sigma\rangle$ and $\left\langle m^{\prime}, \sigma^{\prime}\right\rangle$.

Event 3: $P_{p}$ is corrupt and $P_{b}$ is not corrupt. If $P_{p}$ is corrupt, this shows $\mathscr{A}$ obtains the process of proxy signcryption. On the other hand, $\delta$ can invoke a copy of $\mathscr{A}$; therefore, $\delta$ 
TABLE 1: Comparison of computational efficiency and security.

\begin{tabular}{lcccccc}
\hline Protocols & PKeyGen & ProxyS & Uns & Total & UC security & Semantic security \\
\hline Protocol [4] & $1 \mathrm{H}+1 \mathrm{E}+3 \mathrm{M}+2 \mathrm{P}$ & $2 \mathrm{H}+2 \mathrm{E}+3 \mathrm{M}+2 \mathrm{P}$ & $3 \mathrm{H}+1 \mathrm{E}+1 \mathrm{M}+5 \mathrm{P}$ & $6 \mathrm{H}+4 \mathrm{E}+7 \mathrm{M}+9 \mathrm{P}$ & $\times$ & $\sqrt{ }$ \\
Protocol [19] & $1 \mathrm{H}+1 \mathrm{E}+2 \mathrm{M}+2 \mathrm{P}$ & $3 \mathrm{H}+2 \mathrm{E}+1 \mathrm{M}+2 \mathrm{P}$ & $2 \mathrm{H}+4 \mathrm{E}+6 \mathrm{P}$ & $6 \mathrm{H}+7 \mathrm{E}+3 \mathrm{M}+10 \mathrm{P}$ & $\times$ & $\sqrt{ }$ \\
IBPSP & $1 \mathrm{H}+1 \mathrm{E}+2 \mathrm{M}+2 \mathrm{P}$ & $2 \mathrm{H}+1 \mathrm{E}+3 \mathrm{M}+1 \mathrm{P}$ & $3 \mathrm{H}+1 \mathrm{E}+1 \mathrm{M}+4 \mathrm{P}$ & $6 \mathrm{H}+3 \mathrm{E}+6 \mathrm{M}+7 \mathrm{P}$ & $\sqrt{ }$ & $\sqrt{ }$ \\
\hline
\end{tabular}

can obtain the same information with $\mathscr{A}$. That is to say, when $\mathcal{S}$ makes the proxy signcryption, $\mathcal{S}$ can perfectly simulate $P_{p}$ that is corrupt.

Event 4: $P_{p}$ and $P_{b}$ are both corrupt. In this case, $\mathcal{S}$ can obtain all their input information; i.e., $\mathcal{S}$ can generate real data to simulate the execution of protocol.

In the four events defined above, if $\mathscr{Z}$ can tell whether it interacts with $<\pi_{\text {IBPSP }}, \mathscr{A}>$ or with $<\mathscr{F}$ IBPSP $\mathcal{S}>$ with probability $\left|\operatorname{Pr}\left(\mathscr{Z}^{\left(\pi_{\mathrm{IBPSP}}, \mathscr{A}\right)}\right) \longrightarrow 1-\operatorname{Pr}\left(\mathscr{Z}^{\left(\mathscr{F}_{\mathrm{IBPSP}}, \mathcal{S}\right)}\right) \longrightarrow 1\right|$, then $\mathscr{A}$ wins IND-IBPS-CCA2 with probability $\mid \operatorname{Pr}\left(\mathscr{Z}^{\left(\pi_{\mathrm{IBPSP}}, \mathscr{A}\right)}\right) \longrightarrow$ $1-\operatorname{Pr}\left(\mathscr{Z}^{\left(\mathscr{F}_{\mathrm{IBPSP}}, \mathcal{S}\right)}\right) \longrightarrow 1 \mid$. In the real world, $\operatorname{Pr}\left(\mathscr{Z}^{\left(\pi_{\mathrm{IBPSP}}, \mathscr{A}\right)}\right) \longrightarrow$ 1 is a negligible probability; in addition, $\operatorname{Pr}\left(\mathscr{Z}^{\left(\mathscr{F}_{\text {IBPSP }}, \mathcal{S}\right)}\right) \longrightarrow 1$ is always equal to zero in the ideal world. Therefore, we readily obtain that $\left|\operatorname{Pr}\left(\mathscr{Z}^{\left(\pi_{\text {IBPSP }}, \mathscr{A}\right)}\right) \longrightarrow 1-\operatorname{Pr}\left(\mathscr{Z}^{\left(\mathscr{F}_{\text {IBPSP }}, \mathcal{S}\right)}\right) \longrightarrow 1\right|$ is negligible.

Theorem 10. In the UC framework, $\pi_{I B P S P}$ satisfies the existential unforgeability against adaptive chosen-message attacks.

Proof. Assume there exists a forger. Here we construct an environment $\mathscr{Z}$ and an adversary $\mathscr{A}$ such that, for any adversary $\mathscr{A}, \mathscr{Z}$ cannot tell with nonnegligible probability whether it interacts with $\pi_{\text {IBPSP }}$ and $\mathscr{A}$ in the real-life model or with $\mathscr{F}_{\text {IBPSP }}$ and $\mathcal{S}$ in the ideal model.

$\mathscr{Z}$ proceeds as below. On receiving a request on proxy signcryption of $\mathscr{A}, \mathscr{Z}$ activates $P_{p}$ and outputs the ciphertext $\sigma$ to $\mathscr{A}$. On receiving a request on unsigncryption of $\mathscr{A}, \mathscr{Z}$ activates $P_{b}$ and outputs $\langle m, f>$ to $\mathscr{A}$.

$\mathscr{A}$ proceeds as follows. $\mathscr{A}$ first invokes the forger. As the forger requests to signcrypt a message $m, \mathscr{A}$ requests $\mathscr{Z}$ to signcrypt a message $m$ and outputs the ciphertext $\sigma^{\prime}$ to the forger. On receiving a request on unsigncryption of the forger, $\mathscr{A}$ requests $\mathscr{Z}$ to unsigncrypt $\sigma^{\prime}$ and outputs < $m^{\prime}, f>$ to $\mathscr{A}$ and simultaneously delivers $\sigma^{\prime}$ to the forger. Once the forger receives $m^{\prime}$ and $f=1$, then the forgery $\sigma^{\prime}$ is valid and at this time $\mathscr{Z}$ outputs $f=1$. Clearly, if the forger wins UF-IBPS-CMA-I or UF-IBPS-CMA-I with probability $\varepsilon$, then the forger can succeed in forging valid proxy signcryption. Assume that such a forger exists with nonnegligible probability $\varepsilon$; then the probability that $\mathscr{Z}$ outputs $f=1$ is nonnegligible. However, the probability that $\mathscr{Z}$ outputs $f=1$ is always equal to zero in the ideal model. In other words, if such a forger exists, $\mathscr{Z}$ can always tell with nonnegligible probability whether it interacts with $\pi_{\text {IBPSP }}$ and $\mathscr{A}$ or with $\mathscr{F}_{\text {IBPSP }}$ and $\mathcal{S}$. It contracts with the initial assumption in Theorem 10. As mentioned above, such a forger cannot exist; that is to say, $\pi_{\text {IBPSP }}$ satisfies the existential unforgeability against adaptive chosen-message attacks in the UC framework.

\section{Efficiency Analysis}

In this section, we compare our protocol with similar protocols in terms of computational complexity together with UC security and semantic security (see Table 1). In Table 1, E is one exponent operation, $\mathrm{P}$ is one pairing operation in $G_{2}$, $\mathrm{M}$ is one scalar multiplication operation in $G_{1}$, and $\mathrm{H}$ is one hash operation. Moreover, " $\sqrt{ }$ " means that one cryptography protocol satisfies the relevant security and " $x$ " means that one cryptography protocol does not satisfy the relevant security.

Let $t_{H}$ denote the time cost of one hash operation. According to the literature [21], we can summarize and deduce the time cost of all operations as follows: $\mathrm{P} \approx 1440 t_{H}$, $\mathrm{E} \approx 21 t_{H}, \mathrm{M} \approx 29 t_{H}$, and $\mathrm{H} \approx t_{H}$. We can readily obtain that the time cost of our protocol is $10323 t_{H}$. Following the comparison analysis, there are $13253 t_{H}$ and $14640 t_{H}$ in protocols $[4,19]$, respectively.

From the comparison in Table 1, we obtain that the computation cost of our IBPSP is lower than those of other protocols. It is known from Table 1 that our IBPSP has both semantic security and UC security, but other protocols cannot guarantee the security in the UC framework. All the protocols in Table 1 satisfy the semantic security in the random oracle model.

\section{Summary}

The UC security framework provides the theory basis for the design of protocols in a complex and unpredictable environment. In this paper, we construct an IBPSP with universal composability and prove its semantic security in the random oracle model. We also define the ideal functionality of the IBPSP and prove the equivalence between IBPSP with universal composability and its IND-CCA2 and UF-CMA security. Our IBPSP can guarantee security even when it is composed of an arbitrary set of protocols, or when it is used as a component of an arbitrary system. What is more, our IBPSP can guarantee security even when it concurrently runs with an unbounded number of protocol instances.

\section{Data Availability}

The data used to support the findings of this study are available from the corresponding author upon request.

\section{Conflicts of Interest}

The authors declare that they have no conflicts of interest. 


\section{Acknowledgments}

This work was supported by the Project of Basic Research of Qinghai Province under Grant 2016-ZJ-776 and the Chunhui Project of the Ministry of Education under Grant Z2017052.

\section{References}

[1] M. Mambo, K. Usuda, and E. Okamoto, "Proxy signatures for delegating signing operation," in Proceedings of the 3rd ACM conference on computer and communication security, pp. 48-57, ACM Press, NY, USA, March 1996.

[2] C. Gamage, J. Leiwo, and Y. Zheng, "An efficient scheme foe secure message transmission using proxy-signcryption," in Proceedings of the 22nd Australasim Computer Science Conference, pp. 420-431, Springer, Auckland, 1999.

[3] X. Li and K. Chen, "Identity based proxy-signcryption scheme from pairings," in Proceedings of the Proceedings - IEEE International Conference on Services Computing, (SCC '04), pp. 494497, China, September 2004.

[4] S. X. Chen, S. X. Zhou, X. F. Yao, and F. W. Li, "Efficient identitybased proxy signcryption scheme," Application Research of Computers, vol. 28, no. 7, pp. 2694-2696, 2011.

[5] Y. Ming, J. Feng, and J. Hu Q, "Secure identity-based proxy signcryption scheme in standard model," Journal of Computer Application, vol. 34, no. 10, pp. 2834-2839, 2014.

[6] X. Zhou C, "Identity-based generalized proxy signcryption in the Standard Model," Journal of Cryptologic Research, vol. 3, no. 3, pp. 307-320, 2016.

[7] R. Canetti, "Universally composable security: A new paradigm for cryptographic protocols," in Proceedings of the 42nd IEEE Symposium on Foundation of Computer Science, pp. 136-145, IEEE Computer Society Press, 2001.

[8] R. Canetti, "Universally Composable Signature, Certification, and Authentication," in Proceedings of the 17th Computer Security Foundation Workshop, pp. 219-233, 2004.

[9] Z. Wang, Y. Dai Q, and F. Ye D, "Universally composable identity-based signature," Chinese Journal of electronics, vol. 39, no. 7, pp. 1613-1617, 2011.

[10] Y. L. Tian, J. F. Ma, C. G. Peng, and W. J. Ji, "Universally composable mechanism for group communication," Chinese Journal of Computers. Jisuanji Xuebao, vol. 35, no. 4, pp. 645653, 2012.

[11] Y.-L. Tian, C.-G. Peng, J.-F. Ma, H. Lin, and K. Yang, "Universally composable secure multiparty computation protocol with fairness," Journal on Communication, vol. 35, no. 2, pp. 54-62, 2014.

[12] Z. Zhang, Y. Guo, K. Yang, H. Huang, and Z. Yang, "Universally composable security authenticated key exchange protocol," Journal of Xidian University, vol. 41, no. 5, pp. 185-191, 2014.

[13] B. Yuan Q, Q. Liu J, and Q. Yuan B QLiu J, "A universally composable secure group ownership transfer protocol for tags," Journal of Computer Research, vol. 52, no. 10, pp. 2323-2331, 2015.

[14] C. Zhao, F. H. Yu, and J. M. Li, "Universally composable group blind signature," Application Research of Computers, vol. 34, no. 10, pp. 3109-3111, 2017.

[15] C. Zhao, F. H. Yu, and J. M. Li, "Universally composable group signature protocol," Computer Engineering, vol. 43, no. 3, pp. 172-175, 2017.
[16] M. J. Li, F. H. Yu, and C. Zhao, "Self-certified blind signcryption protocol with UC security, Journal of Frontiers of Computer Science and Technology, vol. 11, no. 6, pp. 932-940, 2017.

[17] X.-X. Hu, Q. H. Zhang, Z. F. Zhang, and F. M. Liu, "Universally composable gateway-oriented-password-authenticated key exchange protocol," Chinese Journal of Computers, vol. 40, no. 5, pp. 1109-1120, 2017.

[18] M. Zhang, Yaom Y., B. Li, and C. Tang, "Accountable mobile e-commerce scheme in intelligent cloud system transactions," Journal of Ambient Intelligence and Humanized Computing, vol. 9, no. 6, pp. 1889-1899, 2018.

[19] J. X. Zhang and Y. M. Wang, "Efficient identity-based proxy signcryption," Computer Engineering and Applications, vol. 43, no. 3, pp. 109-111, 2007.

[20] H.-F. Yu and B. Yang, "Low-computation certificateless hybrid signcryption scheme," Frontiers of Information Technology and Electronic Engineering, vol. 18, no. 7, pp. 928-940, 2017.

[21] C.-I. Fan, W.-Z. Sun, and V. S. Huang, "Provably secure randomized blind signature scheme based on bilinear pairing," Computers \& Mathematics with Applications. An International Journal, vol. 60, no. 2, pp. 285-293, 2010. 


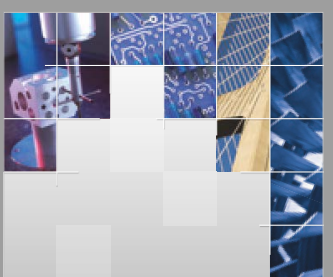

\section{Enfincering}
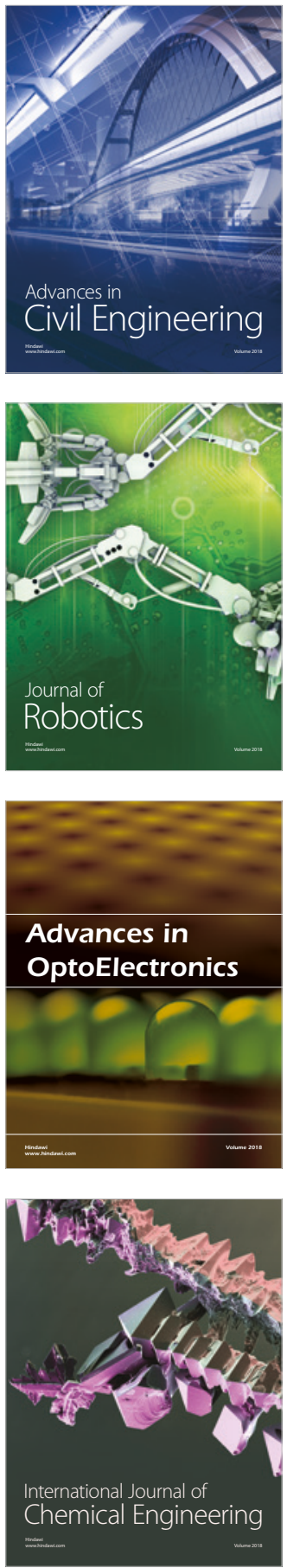

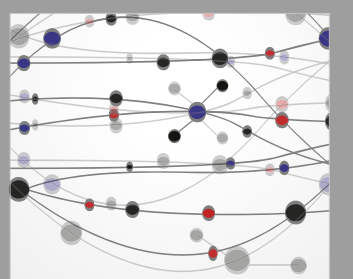

\section{Rotating \\ Machinery}

The Scientific World Journal

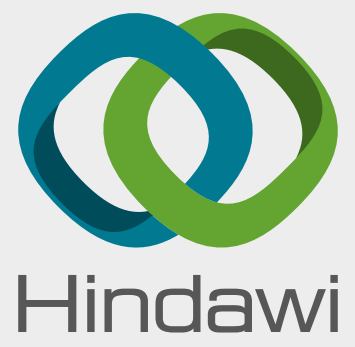

Submit your manuscripts at

www.hindawi.com
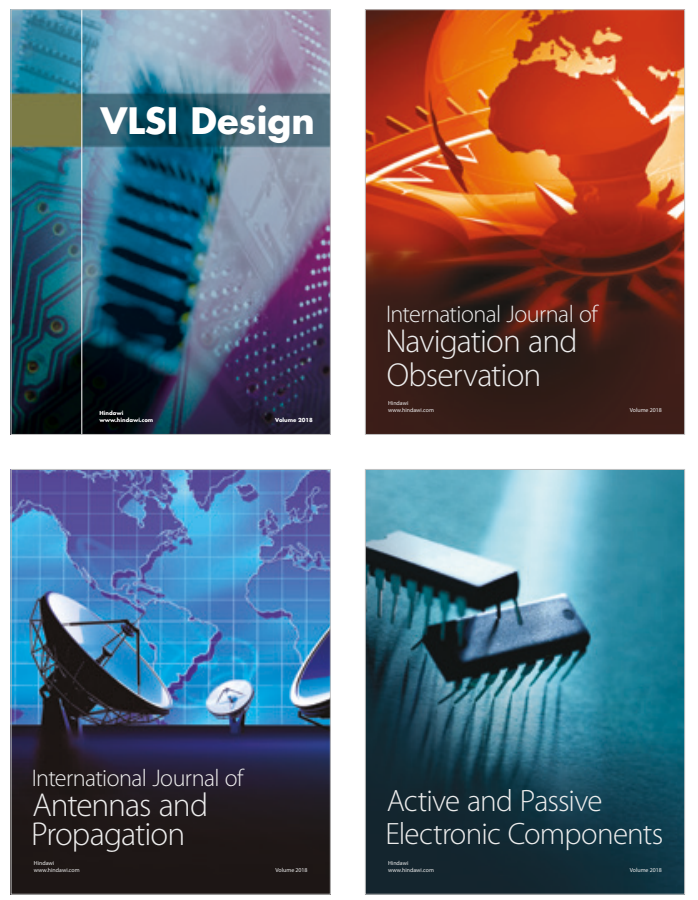
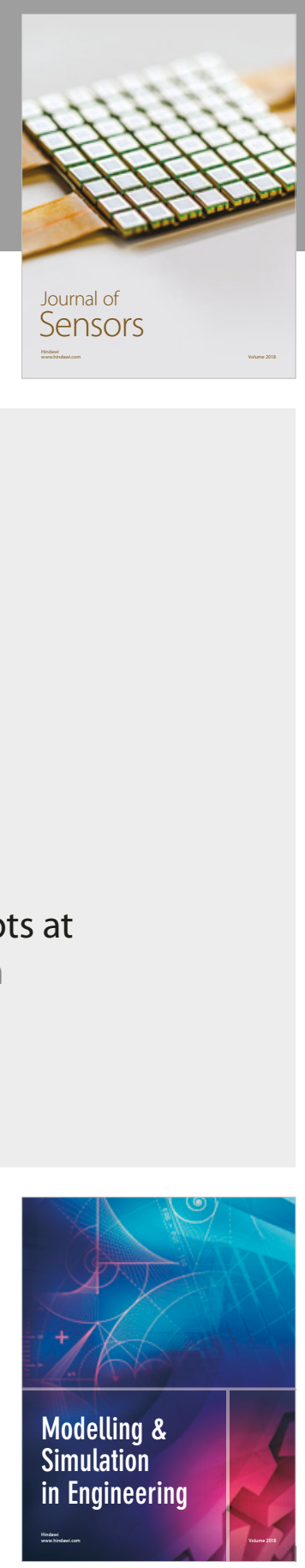

\section{Advances \\ Multimedia}
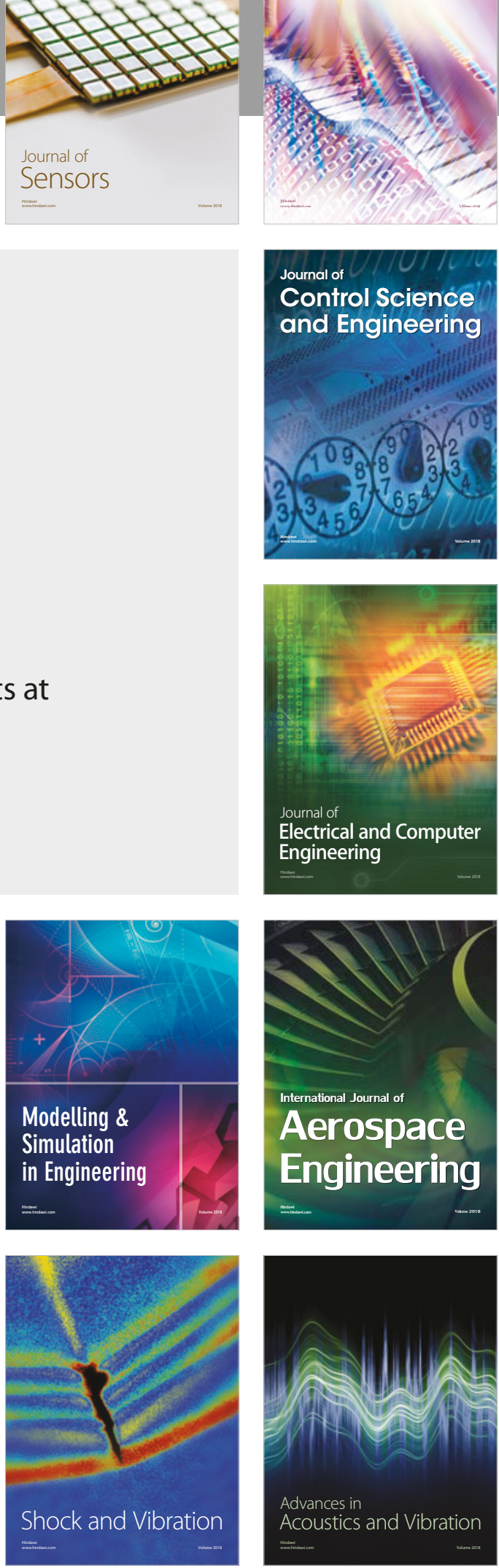